\title{
Does the laparoscopic treatment of paediatric hydroceles represent a better alternative to the traditional open repair technique? A retrospective study of 1332 surgeries performed at two centres in China
}

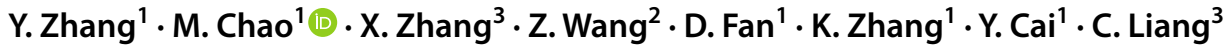

Received: 14 April 2017 / Accepted: 9 December 2017 / Published online: 14 December 2017

(c) The Author(s) 2017. This article is an open access publication

\begin{abstract}
Purpose To evaluate the safety, efficacy and merits of laparoscopic repair in children with hydroceles by comparing the outcomes of laparoscopic repair and the traditional open repair (OR) procedure. The outcomes of the following three laparoscopic percutaneous extra-peritoneal closure (LPEC) approaches were also compared: conventional two-port surgery, transumbilical single-site two-port surgery and single-port surgery.

Methods We retrospectively compared the demographic, perioperative and follow-up data from the consecutive records obtained for 382 boys who underwent OR and 950 boys who underwent LPEC at two children's medical centres in China. In the LPEC group, regardless of the hydrocele form, one of the three approaches with percutaneous aspiration was performed: conventional two-port surgery was performed in 387 cases, single-site two-port surgery was performed in 468 cases and single-port surgery was performed in 95 cases. The clinical data and complications were statistically analysed.

Results Postoperative follow-up data were obtained for all the patients. The mean follow-up time was 36 months (2448 months) in the OR group and 32.5 months (20-44 months) in the LPEC group. Significant differences in recurrence were not observed between the groups (five in the OR and 10 in the LPEC; $P=0.69$ ). However, the operation time, postoperative hospital stay, incidence of scrotal oedema, incision infection and contralateral metachronous hernia or hydrocele were significantly higher in the OR group than those in the LPEC group $(P<0.01)$. Eighteen children $(4.71 \%)$ had a negative exploration of the patent processus vaginalis (PPV) in the OR group. Fourteen children (1.47\%) in the LPEC group had a closed internal ring and were converted to a scrotal procedure. Significant differences in the clinical data or complications were not observed between the two centres for the laparoscopic procedure $(P>0.05)$. Contralateral PPV (cPPV) was found in 18 patients in the single-port group (18.9\%). Of the patients affected with cPPV, significant differences were observed between the single-port group and the two-port LPEC group (122 patients, 31.5\%, $P=0.016)$ and the single-site two-port group (the 148 patients, $31.6 \%, P=0.013$ ). A contralateral metachronous hernia or hydrocele was found in zero, zero and two cases in these groups, respectively, and significant differences were observed $(P<0.01)$ between the single-site surgery and the other two laparoscopic approaches.

Conclusions LPEC is safe, feasible and effective for treating hydroceles in children and has the same recurrence rate as OR. However, LPEC is superior in operation time, hospital stay, occurrence of scrotal oedema, incision infection and occurrence of metachronous hernia or hydrocele. The transumbilical single-site two-port procedure has the same cosmetic effect as the single-port LPEC. According to our experience, the two-port LPEC approach is better for diagnosing cPPV and reducing metachronous hernia or hydrocele than the single-port LPEC procedure.
\end{abstract}

Keywords Laparoscopy $\cdot$ Hydrocele $\cdot$ Hernia needle $\cdot$ Children $\cdot$ Follow-up

M. Chao

cm0654@sina.com

Extended author information available on the last page of the article 


\section{Introduction}

In children, the cause of hydroceles is different from that in adults, and it is mainly caused by the presence of a patent processus vaginalis (PPV) [1]. Traditional open repair (OR) entails performing an inguinal incision, dissecting the inguinal canal, high ligating the PPV [1], and draining the fluid or window created in the tunica vaginalis. With the rapid advancement in medical science, laparoscopic closure of the internal orifice of the PPV has become an option for the treatment of hydroceles in children [2]. The advantages of laparoscopic technology over open surgery include less injury to the spermatic cord and spermatic duct, more aesthetic incisions and the possibility of finding and treating contralateral PPV (cPPV) and other abnormalities [1, 3, 4]. However, the application of laparoscopy in treating hydroceles in children remains controversial. Certain doctors believe that only communicating hydroceles can be treated with this method. Laparoscopy does not affect the peritoneal layer within the scrotum, which may lead to a higher recurrence than OR. In addition, many different laparoscopic surgical modalities are available. To evaluate the safety, feasibility and benefits of the laparoscopic repair of all the forms of paediatric hydroceles and to investigate a better laparoscopic procedure, we conducted this retrospective chart review and report the analysis and summary of relevant experiences.

\section{Patients and methods}

\section{Patients}

In this retrospective chart review, charts from August 2013 to August 2015 for 950 consecutive boys with hydroceles who underwent LPEC at the two professional medical centres that participated in this study were evaluated. The control group included 382 consecutive boys who underwent OR at these centres from January 2012 to December 2012. All patients had a clear diagnosis based on ultrasound-B examinations. Diagnoses included funicular hydrocele, hydrocele testis, spermatic and testicular hydrocele and communicating hydrocele testis. Patients in the LPEC group, regardless of hydrocele forms diagnosed prior to surgery, underwent one of three approaches by different laparoscopic trocar positions: conventional two-port LPEC (two-port LPEC, 387 cases), transumbilical single-site two-port LPEC (singlesite LPEC, 468 cases) or transumbilical single-port LPEC (single-port LPEC, 95 cases).

\section{Surgical techniques}

In the traditional OR group, we performed an inguinal incision and high ligation of the PPV at the level of the internal inguinal ring with drainage of the hydrocele fluid or window creation in the tunica vaginalis. In cases in which a PPV was not probed, we adopted the excisional technique or Jaboulay bottleneck technique [3].

In the LPEC procedure, the patient was placed in a supine position with a monitor at the patient's feet. The surgeon stood on the left side of the patient. A blunted 9-gauge fine injection needle was used as the main puncture device at Centre A (Fig. 1a), and an epidural puncture needle $(80 \times 1.6$ mm, Jiangsu Yang Guang Medical Appliance Co. Ltd. China) was used at Centre B (Fig. 1b). A paediatric hernia hook needle $\left[100 \times 1.6 \mathrm{~mm}\right.$, Surgaid ${ }^{\circledR}$ Medical (XIAMEN) Device Co. Ltd. China] was used for single-port surgery (Fig. 1c).

Establishment of laparoscopic access A 5-mm incision was made through the bottom of the umbilical ring with a Veress needle to establish pneumoperitoneum at a pressure of 6-12 mmHg. A $5 \mathrm{~mm}$ trocar and a $30^{\circ}$ laparoscope were introduced into the peritoneal cavity. A second $3 \mathrm{~mm}$ incision was made for placement of the trocar at the left exterior margin of the rectus abdominis in the conventional two-port LPEC approach (Fig. 2a). In the single-site two-port LPEC
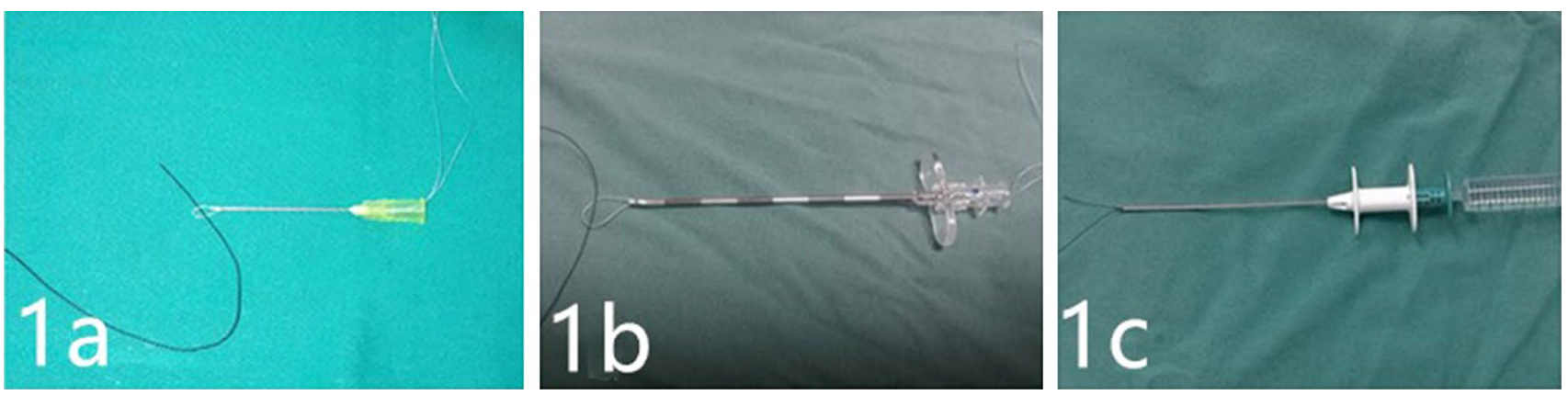

Fig. 1 Three puncture devices: a blunted 9-gauge syringe needle used by the medical centre A; b epidural puncture needle $(80 \times 1.6 \mathrm{~mm}$, Jiangsu Yangguang Medical Appliance Co. Ltd.) used by the medical centre B; and $\mathbf{c}$ paediatric hernia hook needle used in the single-port approach $\left(100 \times 1.6 \mathrm{~mm}\right.$, Surgaid ${ }^{\circledR}$ Medical Device Co. Ltd. $)$ 

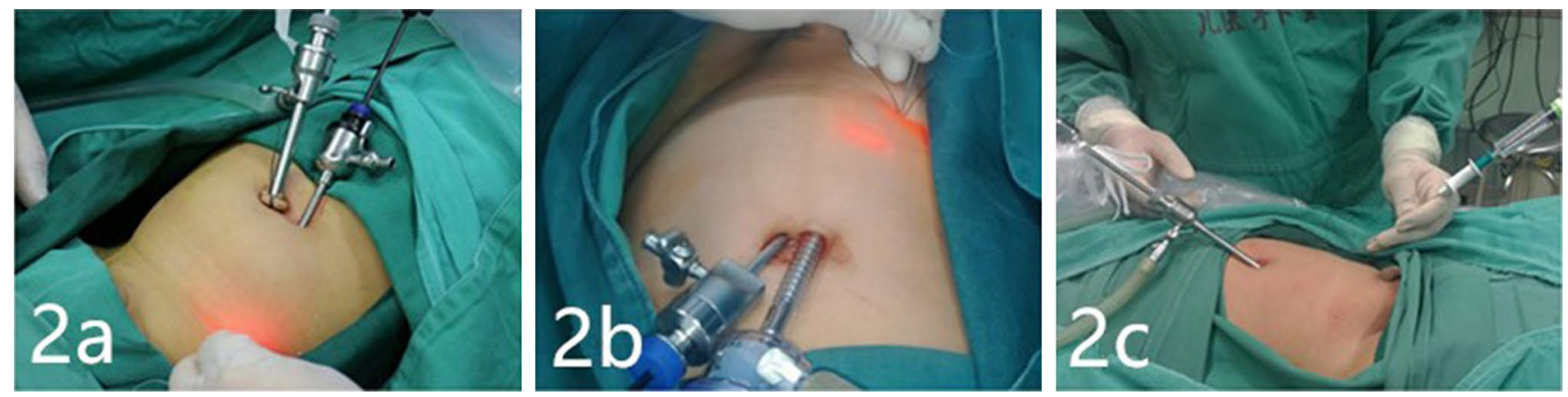

Fig. 2 Position of operation access for the three LPEC approaches: a conventional two-port surgery; and b single-site two-port surgery; and c single-port surgery

approach, a second $3 \mathrm{~mm}$ incision was made on the left side of the umbilical ring for placement of the trocar (Fig. 2b). The single-port LPEC approach via a $5 \mathrm{~mm}$ incision through the bottom of the umbilical ring allowed laparoscope access only (Fig. 2c).

Conventional two-port LPEC approach and single-site two-port LPEC approach A loop of 4-0 braided polyester suture was passed through the tip of a blunted 9-gauge syringe needle or epidural needle and a length of no. 4 or no. 7 non-absorbable surgical silk thread was passed through this loop (Fig. 1a, b). The needle was used to puncture the body surface from the outside at the projected area of the internal ring orifice, with the tip of forceps close to the interior hemicycle of the peritoneum at the internal ring orifice. Under visualization, the needle penetrated the peritoneum lateral to the inferior epigastric vein, the surface of vas deferens and the spermatic cord, leaving one end of the non-absorbable silk thread inside the peritoneal cavity (Fig. 3a). The puncture needle was retracted to just outside the peritoneum but not outside the skin, staying close against the peritoneum along the exterior hemicycle of the internal ring orifice, and a suture was then pulled through the original puncture site in the peritoneum (Fig. 3b). The black silk thread was passed through the braided polyester suture loop with forceps, and then the braided polyester suture loop was tightened and withdrawn from the skin with the silk thread (Fig. 3c). The silk thread was tightened and tied, and the knot was embedded subcutaneously.

Single-port LPEC approach A puncture was made at the projected area on the surface of the internal ring orifice with a no. 4 or 7 non-absorbable suture clipped to a hernia hook needle (Fig. 4a). Under visualization, a small volume of normal saline was injected through a water-injection valve towards the vas deferens and spermatic cord to help puncture the interior of the hemicycle (Fig. 4b). The hook was loosened, and the laparoscope was used to help retain the silk suture within the abdomen, followed by bringing the suture around the exterior of the hemicycle with the help of water injection (Fig. 4c). The silk thread was clipped directly on the hook of the hernia needle, withdrawn from the skin and tied, and the knot was embedded subcutaneously.

Treatment of fluid within the vaginal tunic all the fluid was percutaneously aspirated from the scrotum with $10 \mathrm{~mL}$ syringes after ligation.
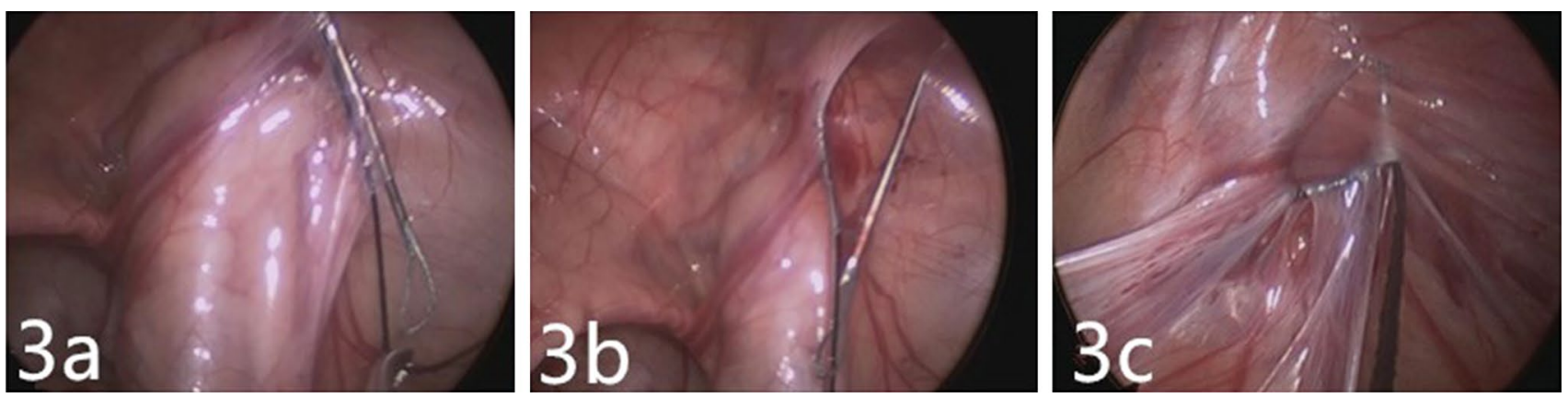

Fig. 3 Conventional two-port LPEC and single-site two-port LPEC: a puncture to the interior inguinal ring; $\mathbf{b}$ puncture exterior to the hemicycle; the black silk thread is passed through the polyester suture loop with forceps; and c the polyester suture loop is tightened and withdrawn through the skin, with the suture knot embedded subcutaneously 

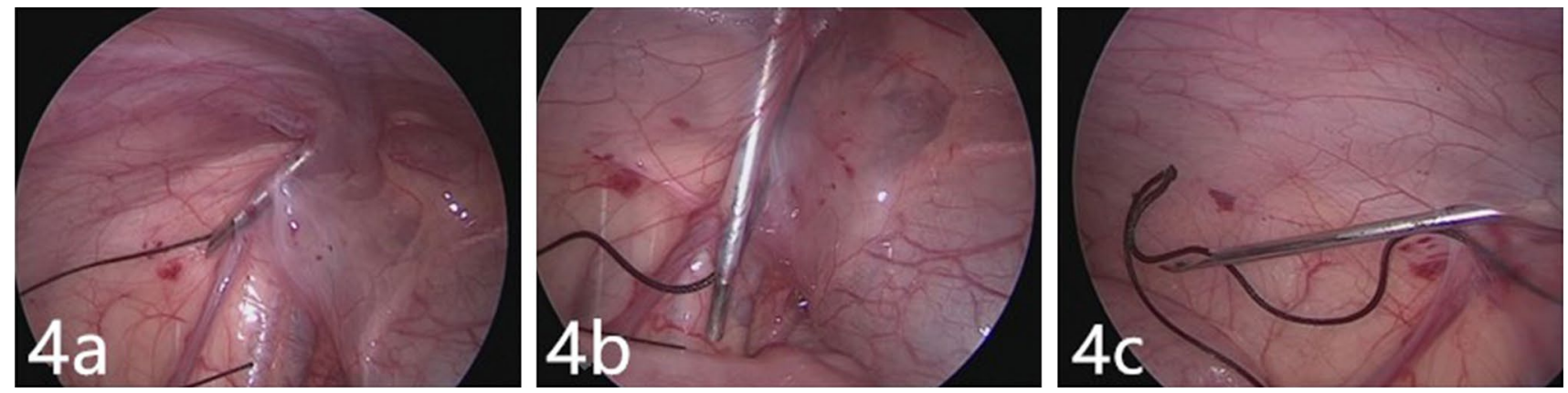

Fig. 4 Puncture technique with hernia needle for single-port approach: a water injection and puncture of the interior of the hemicycle followed by retention of the silk thread in the abdomen; $\mathbf{b}$ water

\section{Follow-up}

Patient visits were arranged 1 month, 6 months and 1 year after surgery for physical examination or ultrasound check. Telephone follow-up examinations were conducted via a conversation with the patients' parents and were arranged by the Telephone Follow-up Centre of Anhui Provincial Children's Hospital before this study.

\section{Statistical analysis}

Information was collected during clinic visits and telephone follow-up examinations, including clinical data and complications in all the cases. Statistical analyses were performed with SPSS 19.0. A $t$ test was used to compare measurement data between the surgical approaches, and one-way ANOVA was used to compare measurement data among the three LPEC groups. A $\chi^{2}$ test was used to compare the rates. $P<0.05$ was considered statistically significant. injection and puncture of the exterior of the hemicycle; and $\mathbf{c}$ the thread pulled out of the body with the hooked needle

\section{Results}

Postoperative follow-up examinations were performed for all the patients. The median follow-up duration after OR was 36 months (range 24-48 months), and follow-up after LPEC was 32.5 months (range 20-44 months). A comparison of the clinical data and complications among the OR versus LPEC patients is shown in Table 1. Significant differences were observed between the OR and LPEC groups in negative PPV exploration [18 (4.71\%) vs. $14(1.47 \%)$, $P<0.01]$, operation time $[28.42 \pm 8.95 \min (10-114)$ vs. $16.11 \pm 12.21 \mathrm{~min}(8-45), P<0.01]$, hospital stay $(2.73 \pm 1.50$ vs. $1.08 \pm 0.31$ days, $P<0.01)$, occurrence of scrotal oedema [71 (18.6\%) vs. $0, P<0.01]$, occurrence of incision infection [6 (1.6\%) vs. $1(0.1 \%), P<0.01]$ and occurrence of contralateral metachronous hernia or hydrocele [7 (1.8\%) vs. $2(0.2 \%), P<0.01]$. Significant differences were not observed between the groups in recurrence [5 (1.3\%) vs. $10(1.1 \%), P=0.69$ ].

Table 1 Comparison of clinical data between traditional open repair (OR) and laparoscopic percutaneous extra-peritoneal closure (LPEC)

\begin{tabular}{|c|c|c|c|c|}
\hline Items & $\begin{array}{l}\text { Traditional open repair } \\
\text { (OR) } \\
N=382\end{array}$ & $\begin{array}{l}\text { Laparoscopic percutaneous extra-peritoneal } \\
\text { closure (LPEC) } \\
N=950\end{array}$ & Statistical value & $P$ value \\
\hline Age (years) & $3.36 \pm 2.32$ & $3.68 \pm 2.20$ & $t=-2.135$ & 0.08 \\
\hline Mean follow-up time (month) & $36(24-48)$ & $32.5(20-44)$ & - & - \\
\hline Recurrence (cases) & $5(1.3 \%)$ & $10(1.1 \%)$ & $\chi^{2}=0.161$ & 0.69 \\
\hline Operation time (min) & $\begin{array}{l}28.42 \pm 8.95 \\
(10-114)\end{array}$ & $\begin{array}{l}16.11 \pm 12.21 \\
(8-45)\end{array}$ & $t=17.11$ & $<0.01$ \\
\hline Hospital stay (days) & $2.73 \pm 1.50$ & $1.08 \pm 0.31$ & $t=21.37$ & $<0.01$ \\
\hline Negative PPV exploration (case) & $18(4.71 \%)$ & $14(1.47 \%)$ & $\chi^{2}=12.18$ & $<0.01$ \\
\hline Scrotal oedema (case) & $71(18.6 \%)$ & 0 & $\chi^{2}=186.5$ & $<0.01$ \\
\hline Incision infection (case) & $6(1.6 \%)$ & $2(0.2 \%)$ & $\chi^{2}=10.27$ & $<0.01$ \\
\hline $\begin{array}{l}\text { Contralateral metachronous hernia or hydro- } \\
\text { celes (case) }\end{array}$ & $7(1.8 \%)$ & $2(0.2 \%)$ & $\chi^{2}=10.68$ & $<0.01$ \\
\hline
\end{tabular}


A comparison between the clinical data and complications among the three LPEC approaches is shown in Table 2. Only 14 patients $(1.47 \%)$ with their internal ring closed during exploration were switched to a trans-scrotal procedure via a minimal incision. None of these 14 patients experienced recurrence during the follow-up period. Significant differences were not observed among the three LPEC approaches in terms of operation time and recurrence $(P>0.05)$. A total of 288 cases (35.8\%) of cPPV were found during LPEC. Statistically significant differences were found between singleport LPEC and the single-site two-port LPEC $(P=0.013)$ and two-port LPEC $(P=0.016)$ in terms of the discovery of cPPV and the occurrence of contralateral metachronous hernia or hydrocele $(P<0.01)$. The umbilical incisions of the single-port and single-site two-port approaches were similar and barely discernible (Fig. 5a, b, c).

Metachronous hydroceles occurred on the contralateral side in two cases after single-port surgery, and reoperation by a transumbilical single-site two-port LPEC confirmed that the non-treated cPPV occurred after the surgery (Fig. 6a). Five OR patients (1.3\%) experienced recurrence. These patients underwent reoperation by open procedure, and each patient had a confirmed reopened or mis-legated PPV. In the LPEC group, ten patients experienced recurrence (1.1\%). Reoperation was performed in five of the 10 cases, which confirmed ligature loosening that resulted in incomplete closure of the PPV (Fig. 6b). The remaining five patients were still under observation at the last follow-up, and two had been treated by trans-scrotal puncture aspiration and remained unvisited again. Significant differences in the recurrence among the three LPEC approaches were not observed.

Each centre performed the LPEC technique using different puncture needles. No significant differences were found between Centre A and Centre B in terms of complications (Table 3).

Table 2 Comparison of general patient data between the three LPEC approaches

\begin{tabular}{|c|c|c|c|c|c|c|}
\hline \multirow[t]{2}{*}{ Items } & \multirow{2}{*}{$\begin{array}{l}\text { All cases } \\
N=950\end{array}$} & \multicolumn{3}{|c|}{ Laparoscopic surgery } & \multirow{2}{*}{$\begin{array}{l}\text { Statistical value } \\
\left(\chi^{2} / \mathrm{F}\right)\end{array}$} & \multirow[t]{2}{*}{$P$ value } \\
\hline & & $\begin{array}{l}\text { Conventional } \\
\text { two-port surgery } \\
N=387\end{array}$ & $\begin{array}{l}\text { Single-site two- } \\
\text { port surgery } \\
N=468\end{array}$ & $\begin{array}{l}\text { Single-port surgery } \\
N=95\end{array}$ & & \\
\hline Age (years) & $3.68 \pm 2.20$ & $4.22 \pm 2.74$ & $2.40 \pm 2.22$ & $3.65 \pm 2.91$ & 2.12 & $0.16^{\mathrm{a}}$ \\
\hline cPPV (case) & $288(35.8 \%)$ & $122(31.5 \%)$ & $148(31.6 \%)$ & $18(18.9 \%)$ & 6.46 & $0.04^{\mathrm{c}^{*}}$ \\
\hline Operation time (min) & $16.11 \pm 12.21(8-45)$ & $21.56 \pm 9.37$ & $17.79 \pm 7.58$ & $22.88 \pm 10.98$ & 5.76 & $0.07^{\mathrm{b}}$ \\
\hline Recurrence (case) & $10(1.1 \%)$ & $4(1.03 \%)$ & $4(0.85 \%)$ & $2(2.10 \%)$ & 1.58 & $0.45^{\mathrm{c}}$ \\
\hline $\begin{array}{l}\text { Contralateral metachronous } \\
\text { hernia/hydrocele (case) }\end{array}$ & $2(0.2 \%)$ & 0 & 0 & $2(2.10 \%)$ & 8.08 & $0.01^{\mathrm{c}}$ \\
\hline
\end{tabular}

* Single-port surgery against conventional two-port surgery $P=0.016$ and against single-site two-port surgery $P=0.013$

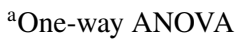

${ }^{\mathrm{b}}$ Nonparametric Kruskal-Wallis rank sum test

${ }^{\mathrm{c}}$ Chi square or Fisher's tests
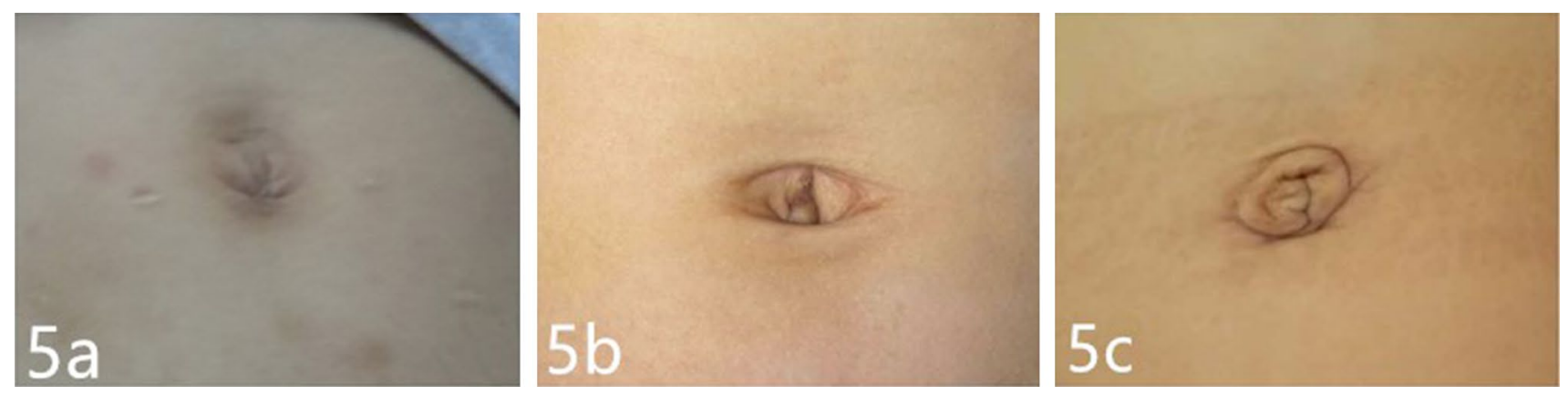

Fig. 5 Photos of the postoperative wound after the three LPEC approaches: a conventional two-port surgery; b single-site two-port surgery; and (c) single-port surgery 
Fig. 6 Residual PPV: a cPPV undiscovered at previous surgery was found during reoperation with the aid of forceps in one case of contralateral metachronous hydrocele after single-port surgery; and b residual PPV with a loosened ligature was found during reoperation in one recurrent case

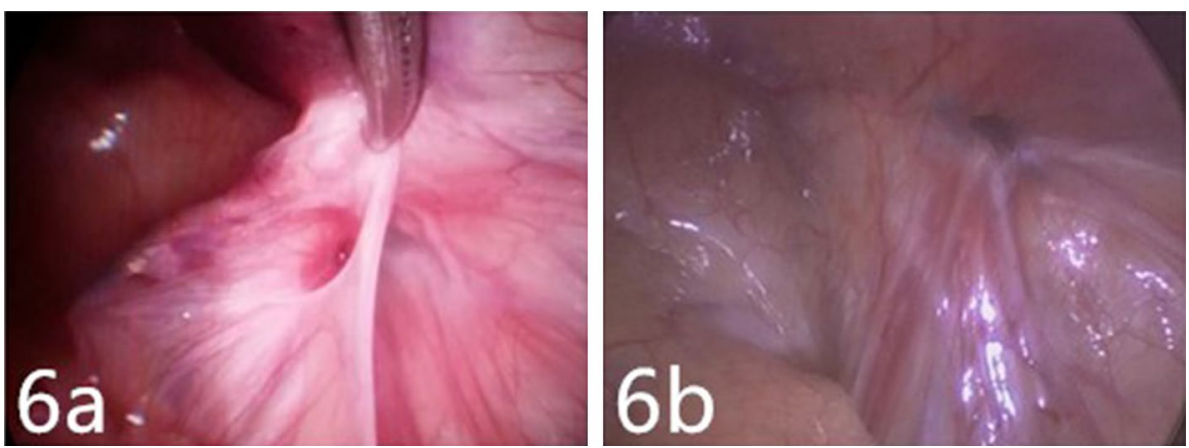

Table 3 Comparison of clinical patient data between the two centres

\begin{tabular}{lllll}
\hline Items & Centre A & Centre B & Statistic al value & $P$ value \\
\hline No. of cases & $\begin{array}{c}628 \text { (unilateral 496; } \\
\text { bilateral 132) }\end{array}$ & $\begin{array}{c}322 \text { (unilateral 309; } \\
\text { bilateral 13) }\end{array}$ & - \\
Age (years) & $3.96 \pm 2.45$ & $4.11 \pm 2.04$ & $t=-0.69$ & 0.49 \\
cPPV (case) & $176(35.4 \%)$ & $112(36.2 \%)$ & $\chi^{2}=0.05$ & 0.88 \\
Open surgery (case) & $8(1.3 \%)$ & $6(1.9 \%)$ & $\chi^{2}=0.51$ & 0.57 \\
Operation time (min) & $22.70 \pm 9.33$ & $17.90 \pm 6.28$ & $t=-1.90$ & 0.07 \\
Recurrence (case) & $(8-45)$ & $(11-23)$ & & 0.51 \\
Umbilical infection (case) & $8(1.3 \%)$ & $2(0.6 \%)$ & $\chi^{2}=0.87$ & $0.55^{*}$ \\
Contralateral metachronous & $2(0.2 \%)$ & $1(0.3 \%)$ & - & 0.55 \\
hernia/hydrocele (case) & & 0 & $\chi^{2}=1.10$ & \\
\hline
\end{tabular}

* Corrected Chi square and Fisher's tests; -: null

\section{Discussion}

Compared with hydrocele pathogenesis in adults, persistent PPV is a common cause of hydroceles in children and explains approximately $60 \%$ of the cases in infants $[1,5]$. The majority of new hydroceles occur after birth and before puberty [3]. In adults, secretions of the tunica vaginalis often cause hydroceles. Therefore, the surgical technique for hydroceles in children differs from that in adults, and good efficacy can be achieved as long as the open PPV is closed [6]. Fluid within the cyst can be removed by aspiration or simply left in place [7]. Because of the changing status of the processus vaginalis, Hussein et al. [8] reported that PPV could spontaneously close in approximately $89 \%$ of infants below 1 year of age. Currently, no studies have specified the exact time at which surgery should be performed in children with hydroceles. Generally, surgical intervention is needed, if the hydroceles persist in children beyond 12-24 months [1, 3]. Higher tensile force may affect blood circulation in the testis, leading to testicular atrophy, and in these cases, surgical treatment is indicated regardless of age [7].

Laparoscopy has been used to treat hydroceles, because the method has been widely applied in inguinal hernia repair in children. The surgical principle of laparoscopic treatment of PPV is the anatomical closure of the PPV $[1,6]$, which is consistent with the treatment of inguinal hernias in children as indicated in previous studies at several centres evaluating the feasibility and safety of the technique [8-12]. Surgeons have stated that laparoscopic surgery is only indicated for communicating hydroceles. However, in a 10-year experience and follow-up report of laparoscopic repair of hydroceles by Yang et al. [6], 283/284 patients $(99.6 \%)$ in their case series were discovered with open internal rings and PPV instead of closed internal rings. In our study, open PPV was found at the internal ring orifice in $98.53 \%$ of patients during laparoscopic surgery and ideal efficacy was achieved following the closure of the internal ring and percutaneous aspiration through the scrotum. With a laparoscopic approach, only 14 patients $(1.47 \%)$ were confirmed to have a negative PPV or internal ring orifice. These patients were switched to the trans-scrotal procedure, which, compared with conventional inguinal OR, resulted in significantly minimized surgical incisions. This was significantly different $(P<0.01)$ to the 18 cases $(4.71 \%)$, who experienced a negative exploration in the OR group, and we regard it as a difficult dissection of the PPV in the open procedure. In comparison to children with inguinal hernias, the peritoneum is usually not thickened and is easier to dissect from the spermatic elements, which makes this procedure feel 
easier than laparoscopic hernia repairs. The comparison of the results of laparoscopic procedures and traditional OR revealed no difference in recurrence between the two procedures. However, regarding the incidence of incision infection, scrotal oedema and contralateral metachronous hernia or hydrocele, the laparoscopic procedure had obvious advantages.

Takehara et al. [13] began successfully using LPEC to treat children with inguinal hernias in 1995. Since then, a succession of modified LPEC techniques have been reported, which differ from each other in the use of LPEC surgical devices, including self-made hernia needles, Endoclose needles, GraNee needles, Reverdin needles, subcutaneous injection needles, common suture needles and epidural needles as suturing instruments [14, 15]. Additionally, an all-in-one optical forceps [16] has been reported as an option of the single-port technique. After repeated trials with different approaches, we concluded that the advantage of a waterfilling hooked hernia needle for paediatric patients is that the surgery can be performed easily with single-port access. Compared with the single-port procedure, the two-port LPEC with a blunted 9-gauge injection needle or epidural needle is inexpensive and presents easy-to-access materials when compared with specific hernia needles, and it has a smaller puncture size, making it more aesthetic than other hernia needles.

The merits of using a secondary forceps are personal preference. First, secondary forceps result in a more rapid and precise completion of ligation, while reducing injury to the spermatic cord and vas deferens and, thus, helps verify the reliability of ligation. Although a suture can also be completed through water injection with a special hernia hook needle, it remains difficult to puncture without the aid of forceps. Second, sometimes a cPPV can only be revealed by the help of forceps, because a peritoneal fold may cover the cPPV. In our study, the cPPV discovery rate was only $18.9 \%$ in the single-port group, which was significantly lower than that in the two-port group (31.5\%) and the single-site group (31.6\%), indicating the importance of forceps when probing cPPV. The use of an all-in-one optical forceps has been reported [16], although this device cannot be widely used for a high number of routine surgeries.

Reducing recurrence remains a major clinical challenge. The most common causes of recurrence are inaccurate ligation or loosening of ligatures $[16,17]$. Not all the recurrences were observed in the early stage of the learning curve, and inaccurate ligation can occur for various reasons, even with experienced doctors. Ten recurrences $(1.1 \%)$ occurred in the 950 LPEC procedures, and this was not significantly different from the five cases that occurred in the OR group (1.3\%). Among the recurrences, all the five cases in the OR group were found as unclosed PPV in reoperations, whereas repetency of the peritoneal orifices was observed in the five recurrent cases in the LPEC groups. As a result, we believe that recurrence was mainly associated with incomplete closure of the PPV and internal ring.

The treatment of cPPV remains controversial $[3,18]$. The reported probability of hernia or hydrocele is approximately $5.6-16 \%$, if cPPV is left untreated [3,18], although substantiated evidence on the cPPV cases that require surgical intervention is not available. Therefore, determining which cPPV case needs to be treated and how to minimize secondary injuries currently remain a major concern. In our study, two cases of contralateral metachronous hernia and hydrocele occurred in the single-port laparoscopic procedure, which was significantly different than that in the OR group (seven cases, 1.8\%) and the other two LPEC groups (zero cases). Consequently, our experience is that all types of cPPV should be treated. Ligation is used to treat cPPV larger than $2 \mathrm{~mm}$, and the peritoneal orifice is torn with forceps for a cPPV diameter less than $2 \mathrm{~mm}$, thus forming a ring-shaped wound surface. Through the observation of recurrence cases during repeat surgery, we have found that the peritoneal orifices treated in this way had already closed completely.

Regarding different surgical approaches, most available studies have tended to favour single-port single-incision laparoscopic treatment for hernias and hydroceles $[1,4,14$, 15]. However, based on our study and experience, we believe that a transumbilical single-site two-port approach is a more feasible technique for the laparoscopic treatment of paediatric hydroceles. No significant difference occurs between the single-site two-port approach and the conventional two-port approach in terms of surgical difficulty or surgical efficacy. With regards to surgical trauma, no adverse event of puncture injury occurred because of application of an additional trocar. The difference in the umbilical wound between single-site two-port surgery and single-port surgery was barely differentiable at 1 year after surgery. The risk of long-term potential for vasal obstruction has yet to be addressed in the literature [3], thus, long-term follow-up is essential to draw the final conclusion on the safety of this technique for the fertility of boys [18].

\section{Conclusions}

LPEC has the same efficacy as traditional OR for the treatment of paediatric hydroceles, but is superior in operation time, hospital stay, occurrence of scrotal oedema, incision infection and occurrence of metachronous hernia or hydrocele. The transumbilical single-site two-port laparoscopic procedure results in healed incisions comparable to the conventional two-port approach, and it has less possibility of metachronous hernia or hydrocele than the single-port approach. 


\section{Compliance with ethical standards}

Conflict of interest YZ, MC, ZR W, DX F, KP Z, YC, CZ L, and XS Z declare that they have no conflicts of interest.

Ethical approval All procedures performed in studies involving human participants were in accordance with the ethical standards of the institutional and/or national research committee and with the 1964 Helsinki declaration and its later amendments or comparable ethical standards.

Human and animal rights This artical is a retrospective chart review, and does not contain any studies with animals performed by any of the authors.

Informed consent All patients signed informed consents when recruited.

Open Access This article is distributed under the terms of the Creative Commons Attribution-NonCommercial 4.0 International License (http://creativecommons.org/licenses/by-nc/4.0/), which permits any noncommercial use, distribution, and reproduction in any medium, provided you give appropriate credit to the original author(s) and the source, provide a link to the Creative Commons license, and indicate if changes were made.

\section{References}

1. Peng Y, Li C, Lin W, Xu L (2015) Application of a laparoscopic, single-port, double-needle technique for pediatric hydroceles with multiple peritoneal folds: a trial from a single-center 5-year experience. Urology 85(6):1466-1470. https://doi.org/10.1016/j. urology.2015.01.053

2. Janetschek G, Reissigl A, Bartsch G (1994) Laparoscopic repair of paediatric hydroceles. J Endourol 8(6):415-417. https://doi. org/10.1089/end.1994.8.415

3. Barthold JS (2012) Abnormalities of the testis and scrotum and their surgical management. In: Wein A, Kavoussi L (eds) CampbellWalsh urology. Elsevier Saunders, Philadelphia, pp 1009-10, 3583

4. Wang Z, Xu L, Chen Z, Chen Z, Yao C, Su Z (2014) Modified single-port minilaparoscopic extraperitoneal repair for paediatric hydrocele: a single-center experience with 279 surgeries. World J Urol 32:1613-1618. https://doi.org/10.1007/s00345-014-1259-8

5. Palmer LS, Rastinehad A (2008) Incidence and concurrent laparoscopic repair of intra-abdominal testis and contralateral patent processus vaginalis. J Urol 72:297-299. https://doi.org/10.1016/j. urology.2007.12.099

6. Yang XD, Wu Y, Xiang B, Wong K, Pei J, Li FY (2015) Ten year experience of laparoscopic repair of paediatric hydrocele and the long-term follow-up results. J Pediatr Surg 50(11):1987-1990. https://doi.org/10.1016/j.jpedsurg.2015.07.007

7. Chengru H (2006) Practical pediatric urology. Version 6. People's Medical Publishing House, Beijing, pp 394-397

8. Naji H, Ingolfsson I, Isacson D, Svensson JF (2012) Decision making in the management of hydroceles in infants and children. Eur J Pediatr 171:807-810. https://doi.org/10.1007/ s00431-011-1628-x

9. Koivusalo AI, Korpela R, Wirtavuori K, Piiparinen S, Rintala RJ, Pakarinen MP (2009) A single-blinded, randomized comparison of laparoscopic versus open hernia repair in children. Pediatrics 123:332-337. https://doi.org/10.1542/peds.2007-3752

10. Chan KL, Hui WC, Tam PKH (2005) Prospective randomized single center, single-blind comparison of laparoscopic vs open repair of paediatric inguinal hernia. Surg Endosc 19:927-932. https://doi.org/10.1007/s00464-004-8224-3

11. Chen H, Bi YL, Lu LIS, Shen J, Tang LF, Liu Y, Zhong HJ (2016) Learning curve of single-incision laparoscopic repair for inguinal hernia and hydrocele in children. J Clin Pediatr Surg 15(04):332 335. https://doi.org/10.3969/j.issn.1671-6353.2016.04.006

12. Chan KL, Chan HY, Tam PK (2007) Towards a near-zero recurrence rate in laparoscopic inguinal hernia repair for paediatric patients of all ages. J Pediatr Surg 42:1993-1997. https://doi. org/10.1016/j.jpedsurg.2007.08.014

13. Takehara H, Yakabe S, Kameoka K (2006) Laparoscopic percutaneous extraperitoneal closure for inguinal hernia in children: clinical outcome of 972 repairs done in 3 paediatric surgical institutions. J Pediatr Surg 41(12):1999-2003. https://doi.org/10.1016/j. jpedsurg.2006.08.032

14. Li SL, Liu L, Yang XF (2014) Status and evaluations of laparoscopic approach in diagnosis and treatment of children with inguinal hernia. Chin J Pediatr Surg 35(6):406-409. https://doi. org/10.3760/cma.j.issn.0253-3006.2014.06.002

15. Liu W, Wu R, Du G (2014) Single-port laparoscopic extraperitoneal repair of paediatric inguinal hernias and hydroceles by using modified Kirschner pin: a novel technique. Hernia 18(3):345-349. https://doi.org/10.1007/s10029-013-1181-9

16. Yilmaz E, Afsarlar CE, Senel E, Cavusoglu YH, Karaman I, Karaman A, Ozguner IF (2015) A novel technique for laparoscopic inguinal hernia repair in children: single-port laparoscopic percutaneous extraperitoneal closure assisted by an optical forceps. Pediatr Surg Int 31(7):639-646. https://doi.org/10.1007/ s00383-015-3722-z

17. Saranga Bharathi R, Arora M, Baskaran V (2008) Minimal access surgery of paediatric inguinal hernias: a review. Surg Endosc 22(8):1751-1762. https://doi.org/10.1007/s00464-008-9846-7

18. Montupet P, Esposito C (2011) Fifteen year experience in laparoscopic inguinal hernia repair in paediatric patients. Results and considerations on a debated procedure. Surg Endosc 25(2):450 453. https://doi.org/10.1007/s00464-010-1188-6

\title{
Affiliations
}

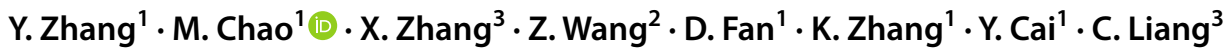 \\ Y. Zhang \\ zhangyincx@163.com \\ X. Zhang \\ xiansheng-zhang@163.com \\ Z. Wang \\ ahsetyymnk@163.com \\ D. Fan \\ ahsetyymnwk@126.com \\ K. Zhang \\ zkp306@sina.com \\ Y. Cai \\ caiying2506@163.com
}




\section{Liang \\ 39211144@qq.com}

1 Department of Urology, Anhui Provincial Children's Hospital, No. 39, East Wangjiang Road, Hefei 230051, Anhui, China
2 Department of Pediatric Surgery, Anhui Provincial Hospital, Anhui Medical University, Hefei 230001, Anhui, China

3 Department of Urology, The First Affiliated Hospital of Anhui Medical University, Hefei 230022, Anhui, China 